12

\title{
Влияние ограниченной апертуры излучателя на характеристики ширины и угла расходимости лазерного пучка
}

\author{
(C) А.М. Райцин \\ Всероссийский научно-исследовательский институт оптико-физических измерений, \\ 119361 Москва, Россия \\ e-mail: arcadiyram@rambler.ru
}

Поступила в редакцию 30.04.2019 г.

В окончательной редакции 05.07.2019 г.

Принята к публикации 10.07.2019 г.

\begin{abstract}
Определены необходимые условия для корректного измерения ширины и угла расходимости лазерного пучка с учетом конечных размеров апертуры излучателя методом, основанным на определении начальных пространственных моментов. Показано, что в этом случае предъявляются жесткие условия к форме пространственного распределения интенсивности в поперечном сечении лазерного пучка, которые не принимались во внимание в соответствующих разработанных стандартах.
\end{abstract}

Ключевые слова: ширина, диаметр, расходимость, коэффициент распространения, пространственное распределение интенсивности лазерного пучка.

DOI: 10.21883/OS.2019.11.48527.149-19

\section{Введение}

В настоящее время основными пространственно-энергетическими характеристиками лазерного пучка являются ширина и угол расходимости, регламентированные международным стандартом еще в начале 2000-х годов, основанном на измерении начальных пространственных моментов [1].

Данные характеристики определяют также коэффициент распространения пучка $M^{2}$. В процессе обсуждения этих характеристик в [2] было обращено внимание на то, что они имеют конечные разумные значения лишь при выполнении жестких условий, накладываемых на форму распределения поля.

Сами эти жесткие условия не были строго сформулированы, но отмечено, что при наличии разрывов в зависимости интенсивности от поперечных координат вторые моменты углового распределения, связанные с параметром $M^{2}$, шириной и углом расходимости, становятся неограниченно большими. В качестве альтернативных пространственно-энергетических характеристик лазерного пучка известны также диаметр, угловая и энергетическая расходимости, определяемые по заданному уровню интенсивности или энергии, не имеющие такого недостатка. Для их измерения ранее применялся метод двух сечений, основанный на линейной зависимости диаметра лазерного пучка от расстояния [3].

В [4] была впервые предпринята попытка характеризовать лазерное излучение на языке пространственных моментов и показан параболический характер зависимостей вторых моментов распределения интенсивности (РИ) от расстояния (или гиперболический характер ширины пучка от расстояния), что позволило отказаться от метода двух сечений, считая данные зависимости более точным приближением. В результате характеристики ширины, угла расходимости лазерного пучка, устанавливаемые в [1], опираются на гиперболическую зависимость ширины пучка от расстояния.

Так, для гаусс-эллиптического РИ, главные оси которого ориентированы по осям координат $x, y$, зависимость ширин пучка от расстояния $z$ определяется по формулам

$$
\begin{aligned}
& d_{x}(z)=4 \sqrt{m_{20}(z) / m_{00}-\left(m_{10}(z) / m_{00}\right)^{2}}, \\
& d_{y}(z)=4 \sqrt{m_{02}(z) / m_{00}-\left(m_{01}(z) / m_{00}\right)^{2}},
\end{aligned}
$$

где $m_{00}=m_{00}(0)$, a $m_{20}(z), m_{02}(z), m_{10}(z), m_{01}(z)-$ пространственные моменты РИ.

Заметим, что основой рассуждений, приводящих к данной зависимости, является уравнение квазиоптики, их которого вытекает известная связь между комплексной амплитудой поля в поперечном сечении пучка $U(x, y, z)$ с координатой $z$ с комплексной амплитудой поля в плоскости излучателя $u\left(x_{1}, y_{1}, 0\right)$ [5]

$$
\begin{aligned}
& U(x, y, z)=\frac{\exp (i 2 \pi z / \lambda)}{i \lambda z} \int_{-\infty}^{\infty} \int_{-\infty}^{\infty} u\left(x_{1}, y_{1}, 0\right) \\
& \quad \times \exp \left(i \frac{\pi}{\lambda z}\left(\left(x-x_{1}\right)^{2}+\left(y-y_{1}\right)^{2}\right)\right) d x_{1} d y_{1}
\end{aligned}
$$

где $\lambda$ - длина волны излучения.

Строго говоря, при выводе (1) использовалось предположение об ограниченных поперечных размерах источника и замена конечных пределов интегрирования бесконечными по всей плоскости расположения излучателя является допущением, требующим уточнения. 
Для этого предполагается, что модуль амплитуды поля источника за пределами апертуры излучателя равен нулю. В пределах апертуры амплитуда поля может изменяться различным образом и ее значение на границе не обязательно равно нулю.

Например, в случае гауссова лазерного пучка модуль его амплитуды в направлении границ быстро убывает и достигает на границе малого значения, которое часто заменяется нулевым, что является приближением, обычно не приводящим к противоречиям при измерении РИ [6].

Однако при измерении параметров пучка, основанных на начальных моментах, упомянутое приближение существенно и необходимо определить условия их существования при конечных размерах апертуры излучателя, что является целью настоящей работы. Показано, что для всех непрерывных РИ, им не удовлетворяющих, вторые моменты РИ являются расходящимися.

Это обстоятельство ставит вопросы к корректному применению коэффициента распространения, ширины и угла расходимости в качестве стандартизованных характеристик лазерного пучка.

\section{Постановка задачи}

Распределение интенсивности $I(x, y, z) \geq 0$ в поперечном сечении лазерного пучка на расстоянии $z>0$ от квадратной апертуры излучателя с линейным размером $T$ равно

$$
I(x, y, z)=U(x, y, z) U^{*}(x, y, z),
$$

где $U(x, y, z)$ определяется выражением (1), в котором пределы интегрирования ограничены промежутком $(-T / 2 ; T / 2), *-$ знак комплексного сопряжения.

Будем считать функцию $u\left(x_{1}, y_{1}, 0\right)$ на выбранном промежутке непрерывной и дважды дифференцируемой.

С учетом (1) и (2) выражение для РИ запишем в виде

$$
\begin{aligned}
& I(x, y, z)=\frac{1}{\lambda^{2} z^{2}} \int_{-T / 2}^{T / 2} \int_{-T / 2}^{T / 2} \int_{-T / 2}^{T / 2} \int_{-T / 2}^{T / 2} u\left(x_{1}, y_{1}, 0\right) \\
& \quad \times u^{*}\left(x_{2}, y_{2}, 0\right) \exp \left(i \frac{\pi}{\lambda z}\left(x_{1}-x_{2}\right)\left(x_{1}+x_{2}-2 x\right)\right) \\
& \quad \times \exp \left(i \frac{\pi}{\lambda z}\left(y_{1}-y_{2}\right)\left(y_{1}+y_{2}-2 y\right)\right) d x_{1} d x_{2} d y_{1} d y_{2} .
\end{aligned}
$$

Нас будет интересовать зависимость от $z$ нормированных начальных пространственных моментов РИ, представляющих отношение несобственных интегралов

$$
m_{k l}(z)=\frac{\int_{-\infty}^{\infty} \int_{-\infty}^{\infty} x^{k} y^{l} I(x, y, z) d x d y}{\int_{-\infty}^{\infty} \int_{-\infty}^{\infty} I(x, y, z) d x d y}, \quad k, l=0,1,2 .
$$

и определение условий их сходимости.

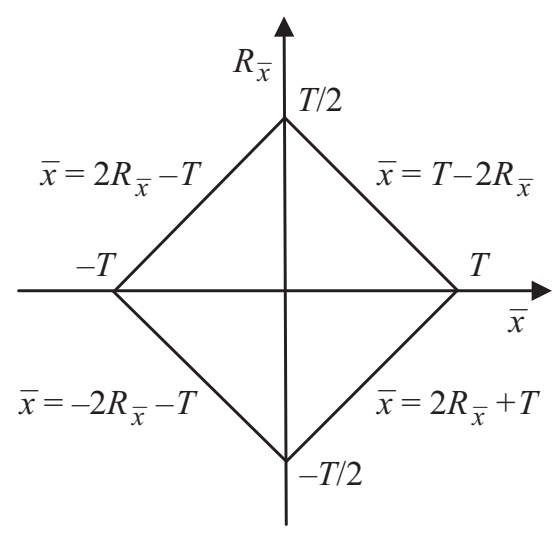

Рис. 1. Области интегрирования.

\section{Вычисление нормированных начальных пространственных моментов}

Для решения задачи введем в (3) новые переменные интегрирования

$$
\begin{array}{ll}
\bar{x}=x_{1}-x_{2} ; & R_{\bar{x}}=\left(x_{1}+x_{2}\right) / 2 ; \\
\bar{y}=y_{1}-y_{2} ; & R_{\bar{y}}=\left(y_{1}+y_{2}\right) / 2 .
\end{array}
$$

Тогда

$$
\begin{array}{ll}
x_{1}=R_{\bar{x}}+\frac{\bar{x}}{2} ; & y_{1}=R_{\bar{y}}+\frac{\bar{y}}{2}, \\
x_{2}=R_{\bar{x}}-\frac{\bar{x}}{2} ; & y_{2}=R_{\bar{y}}-\frac{\bar{y}}{2} .
\end{array}
$$

На рис. 1 представлена область интегрирования в новых переменных $\bar{x}, R_{\bar{x}}$. Аналогично изменится область интегрирования и для переменных $\bar{y}, R_{\bar{y}}$.

После замены переменных выражение (3) можно записать в виде

$$
\begin{aligned}
& I(x, y, z)=\frac{1}{\lambda^{2} z^{2}} \int_{0}^{T / 2} d R_{\bar{y}} \\
& \times \int_{-\left(T-2 R_{\bar{y}}\right)}^{T-2 R_{\bar{y}}}\left[\bar{I}\left(x, \bar{y},-R_{\bar{y}}, z\right) \exp \left(-i \frac{2 \pi}{\lambda z} \bar{y}\left(R_{\bar{y}}+y\right)\right)\right. \\
& \left.+\bar{I}\left(x, \bar{y}, R_{\bar{y}}, z\right) \exp \left(i \frac{2 \pi}{\lambda z} \bar{y}\left(R_{\bar{y}}-y\right)\right)\right] d \bar{y},
\end{aligned}
$$

где

$$
\begin{gathered}
\bar{I}\left(x, \bar{y}, R_{\bar{y}}, z\right)=\int_{0}^{T / 2} d R_{\bar{x}} \int_{-\left(T-2 R_{\bar{x}}\right)}^{T-2 R_{\bar{x}}}\left(\mu\left(x,-R_{\bar{x}}, \bar{x}, R_{\bar{y}}, \bar{y}, z\right)\right. \\
\left.+\mu\left(x, R_{\bar{x}}, \bar{x}, R_{\bar{y}}, \bar{y}, z\right)\right) d \bar{x}, \\
\mu\left(x, R_{\bar{x}}, \bar{x}, R_{\bar{y}}, \bar{y}, z\right)=u\left(R_{\bar{x}}+\frac{\bar{x}}{2}, R_{\bar{y}}+\frac{\bar{y}}{2}, 0\right) \\
\quad \times u^{*}\left(R_{\bar{x}}-\frac{\bar{x}}{2}, R_{\bar{y}}-\frac{\bar{y}}{2}, 0\right) \exp \left(i \frac{2 \pi}{\lambda z} \bar{x}\left(R_{\bar{x}}-x\right)\right) .
\end{gathered}
$$


Рассмотрим начальный момент $m_{20}(z)$. Тогда

$$
\begin{aligned}
m_{20}(z) & =\frac{J_{2}(z)}{J_{0}(z)}=\frac{\int_{-\infty}^{\infty} x^{2} d x \int_{-\infty}^{\infty} I(x, y, z) d y}{\int_{-\infty}^{\infty} \int_{-\infty}^{\infty} I(x, y, z) d x d y} \\
= & \frac{\lim _{L \rightarrow \infty} \int_{-L}^{L} x^{2} d x \int_{-L}^{L} I(x, y, z) d y}{\lim _{L \rightarrow \infty} \int_{-L}^{L} d x \int_{-L}^{L} I(x, y, z) d y} .
\end{aligned}
$$

С учетом (4) интеграл $\int_{-L}^{L} I(x, y, z) d y$, входящий в числитель (5), равен

$$
\begin{aligned}
& \int_{-L}^{L} I(x, y, z) d y=\frac{1}{\lambda^{2} z^{2}} \int_{0}^{T / 2} d R_{\bar{y}} \int_{-\left(T-2 R_{\bar{y}}\right)}^{T-2 R_{\bar{y}}}\left[\bar{I}\left(x, \bar{y},-R_{\bar{y}}, z\right)\right. \\
& \left.\times \exp \left(-i \frac{2 \pi}{\lambda z} \bar{y} R_{\bar{y}}\right)+\bar{I}\left(x, \bar{y}, R_{\bar{y}}, z\right) \exp \left(i \frac{2 \pi}{\lambda z} \bar{y} R_{\bar{y}}\right)\right] \\
& \times \int_{-L}^{L} \exp \left(-i \frac{2 \pi}{\lambda z} \bar{y} y\right) d y d \bar{y} .
\end{aligned}
$$

Интегрируя (6) по $y$, получим

$$
\begin{gathered}
\int_{-L}^{L} I(x, y, z) d y=\frac{1}{\lambda z} \int_{0}^{T / 2} d R_{\bar{y}} \int_{-\left(T-2 R_{\bar{y}}\right)}^{T-2 R_{\bar{y}}}\left[\bar{I}\left(x, \bar{y},-R_{\bar{y}}, z\right)\right. \\
\times \exp \left(-i \frac{2 \pi}{\lambda z} \bar{y} R_{\bar{y}}\right)+\bar{I}\left(x, \bar{y}, R_{\bar{y}}, z\right) \\
\left.\times \exp \left(i \frac{2 \pi}{\lambda z} \bar{y} R_{\bar{y}}\right)\right] \psi(\bar{y}, L) d \bar{y} \\
\psi(\bar{y}, L)=\frac{1}{\pi \bar{y}} \sin \left(\frac{2 \pi \bar{y} L}{\lambda z}\right) .
\end{gathered}
$$

Тогда $J_{2}(z)$ можно записать в виде

$$
\begin{aligned}
& J_{2}(z)=\frac{1}{\lambda z} \int_{0}^{T / 2} d R_{\bar{y}}\left(\lim _{L \rightarrow \infty} \int_{-\left(T-2 R_{\bar{y}}\right)}^{T-2 R_{\bar{y}}} \psi(\bar{y}, L) d \bar{y}\right. \\
& \times \int_{-L}^{L} x^{2}\left[\bar{I}\left(x, \bar{y},-R_{\bar{y}}, z\right) \exp \left(-i \frac{2 \pi}{\lambda z} \bar{y} R_{\bar{y}}\right)\right. \\
& \left.\left.+\bar{I}\left(x, \bar{y}, R_{\bar{y}}, z\right) \exp \left(i \frac{2 \pi}{\lambda z} \bar{y} R_{\bar{y}}\right)\right] d x\right) .
\end{aligned}
$$

Так как $\lim _{L \rightarrow \infty} \psi(\bar{y}, L)=\lim _{L \rightarrow \infty}\left(\frac{1}{\pi \bar{y}} \sin \left(\frac{2 \pi \bar{y} L}{\lambda z}\right)\right)=\delta(\bar{y})$, где $\delta(\bar{y})$ - дельта-функция, для (7) получим

$$
J_{2}(z)=\frac{1}{\lambda z} \int_{-T / 2}^{T / 2}\left(\lim _{L \rightarrow \infty} \int_{-L}^{L} x^{2} \bar{I}\left(x, 0, R_{\bar{y}}, z\right) d x\right) d R_{\bar{y}},
$$

где

$$
\begin{gathered}
\bar{I}\left(x, 0, R_{\bar{y}}, z\right)=\int_{0}^{T / 2} d R_{\bar{x}} \int_{-\left(T-2 R_{\bar{x}}\right)}^{T-2 R_{\bar{x}}} \exp \left(-i \frac{2 \pi}{\lambda z} \bar{x} x\right) \\
\times\left(\bar{\mu}\left(-R_{\bar{x}}, \bar{x}, R_{\bar{y}}, z\right)+\bar{\mu}\left(R_{\bar{x}}, \bar{x}, R_{\bar{y}}, z\right)\right) d \bar{x} \\
\bar{\mu}\left(R_{\bar{x}}, \bar{x}, R_{\bar{y}}, z\right)=u\left(R_{\bar{x}}+\frac{\bar{x}}{2}, R_{\bar{y}}, 0\right) \\
\times u^{*}\left(R_{\bar{x}}-\frac{\bar{x}}{2}, R_{\bar{y}}, 0\right) \exp \left(i \frac{2 \pi}{\lambda z} \bar{x} R_{\bar{x}}\right) .
\end{gathered}
$$

Интегрируя (8) по переменной $x$ и учитывая, что

$$
\begin{gathered}
\int_{-L}^{L} x^{2} \exp \left(-i \frac{2 \pi}{\lambda z} \bar{x} x\right) d x=-\frac{\lambda^{3} z^{3}}{4 \pi^{2}} \psi_{\bar{x}}^{\prime \prime}(\bar{x}, L), \\
\psi(\bar{x}, L)=\frac{1}{\pi \bar{x}} \sin \left(\frac{2 \pi \bar{x} L}{\lambda z}\right)
\end{gathered}
$$

запишем для $J_{2}(z)$

$$
\begin{aligned}
& J_{2}(z)=\frac{-\lambda^{2} z^{2}}{4 \pi^{2}} \int_{-T / 2}^{T / 2} d R_{\bar{y}}\left(\lim _{L \rightarrow \infty} \int_{0}^{T / 2} d R_{\bar{x}}\right. \\
& \left.\times \int_{-\left(T-2 R_{\bar{x}}\right)}^{T-2 R_{\bar{x}}}\left(\bar{\mu}\left(-R_{\bar{x}}, \bar{x}, R_{\bar{y}}, z\right)+\bar{\mu}\left(R_{\bar{x}}, \bar{x}, R_{\bar{y}}, z\right)\right) \psi_{\bar{x}}^{\prime \prime}(\bar{x}, L) d \bar{x}\right) .
\end{aligned}
$$

Последовательно интегрируя (9) по частям и учитывая, что $\psi_{\bar{x}}^{\prime}(-\bar{x}, L)=-\psi_{\bar{x}}^{\prime}(\bar{x}, L), \lim _{L \rightarrow \infty} \psi(\bar{x}, L)=\delta(\bar{x})$, после громоздких преобразований получим

$$
\begin{aligned}
& J_{2}(z)=\frac{-\lambda^{2} z^{2}}{4 \pi^{2}} \int_{-T / 2}^{T / 2} d R_{\bar{y}}\left[-\lim _{L \rightarrow \infty}\left(I\left(-T / 2, R_{\bar{y}}, 0\right)\right.\right. \\
& \left.\left.+I\left(T / 2, R_{\bar{y}}, 0\right)\right) \psi(0, L)+\int_{-T / 2}^{T / 2} \bar{\mu}^{\prime \prime}\left(R_{\bar{x}}, R_{\bar{y}}, z\right) d R_{\bar{x}}\right]
\end{aligned}
$$

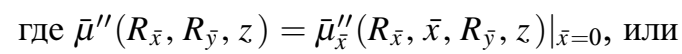

$$
\begin{aligned}
\bar{\mu}^{\prime \prime}\left(R_{\bar{x}}, R_{\bar{y}}, z\right)= & {\left[u\left(R_{\bar{x}}+\frac{\bar{x}}{2}, R_{\bar{y}}, 0\right) u^{*}\left(R_{\bar{x}}-\frac{\bar{x}}{2}, R_{\bar{y}}, 0\right)\right.} \\
& \left.\times \exp \left(i \frac{2 \pi}{\lambda z} \bar{x} R_{\bar{x}}\right)\right]\left._{\bar{x}}^{\prime \prime}\right|_{\bar{x}=0} \cdot
\end{aligned}
$$


Из (10) следует, что

$$
\begin{aligned}
& \lim _{L \rightarrow \infty}\left(I\left(-T / 2, R_{\bar{y}}, 0\right)+I\left(T / 2, R_{\bar{y}}, 0\right)\right) \psi(0, L) \\
& =\delta(0)\left(I\left(-T / 2, R_{\bar{y}}, 0\right)+I\left(T / 2, R_{\bar{y}}, 0\right)\right) .
\end{aligned}
$$

Так как $\lim \psi(0, L)=\delta(0)=\infty ; \quad I(x, y, z) \geq 0$, то предел существует, если

$$
I\left(-T / 2, R_{\bar{y}}, 0\right)=I\left(T / 2, R_{\bar{y}}, 0\right)=0,
$$

т.е. интенсивность на границах апертуры в плоскости излучателя должна равняться нулю.

Результат справедлив также для рассматриваемой в [4] бесконечной апертуры, т. е. если

$$
\lim _{T \rightarrow \infty} I\left(-T / 2, R_{\bar{y}}, 0\right)=\lim _{T \rightarrow \infty} I\left(T / 2, R_{\bar{y}}, 0\right)=0 .
$$

При выполнении (12)

$$
J_{2}(z)=\frac{-\lambda^{2} z^{2}}{4 \pi^{2}} \int_{-T / 2}^{T / 2} \int_{-T / 2}^{T / 2} \bar{\mu}^{\prime \prime}\left(R_{\bar{x}}, R_{\bar{y}}, z\right) d R_{\bar{x}} d R_{\bar{y}}
$$

Подставляя (11) в (13), и заменяя $R_{\bar{x}}=x ; R_{\bar{y}}=y$, получим

$$
\begin{aligned}
& J_{2}(z)=\frac{-\lambda^{2} z^{2}}{16 \pi^{2}} \int_{-T / 2}^{T / 2} \int_{-T / 2}^{T / 2}\left(\frac{\partial u^{2}(x, y, 0)}{\partial x^{2}} u^{*}(x, y, 0)\right. \\
& -2 \frac{\partial u(x, y, 0)}{\partial x} \frac{\partial u^{*}(x, y, 0)}{\partial x} \\
& \left.+u(x, y, 0) \frac{\partial^{2} u^{*}(x, y, 0)}{\partial x^{2}}\right) d x d y \\
& +\int_{-T / 2}^{T / 2} \int_{-T / 2}^{T / 2} x^{2} I(x, y, 0) d x d y \\
& +\frac{i \lambda z}{2 \pi} \int_{-T / 2}^{T / 2} \int_{-T / 2}^{T / 2} x\left(u(x, y, 0) \frac{\partial u^{*}(x, y, 0)}{\partial x}\right. \\
& \left.-u^{*}(x, y, 0) \frac{\partial u(x, y, 0)}{\partial x}\right) d x d y
\end{aligned}
$$

где $I(x, y, 0)=u(x, y, 0) u^{*}(x, y, 0)$.

Аналогично можно получить выражение для знаменателя формулы (5)

$$
J_{0}(z)=J_{0}(0)=\int_{-T / 2}^{T / 2} \int_{-T / 2}^{T / 2} I(x, y, 0) d x d y .
$$

Для применения формул (14) и (15) удобно комплексные амплитуды $u(x, y, 0), u^{*}(x, y, 0)$ представить в виде

$$
\begin{aligned}
u(x, y, 0) & =|u(x, y, 0)| e^{i \varphi(x, y, 0)}, \\
u^{*}(x, y, 0) & =|u(x, y, 0)| e^{-i \varphi(x, y, 0)} .
\end{aligned}
$$

Подставляя (16) в (14), после упрощений получим

$$
\begin{aligned}
& J_{2}(z)=\frac{\lambda^{2} z^{2}}{4 \pi^{2}} \int_{-T / 2}^{T / 2} \int_{-T / 2}^{T / 2} \frac{1}{2}\left(-|u(x, y, 0)| \cdot|u(x, y, 0)|_{x}^{\prime \prime}\right. \\
& \left.+2|u(x, y, 0)|^{2}\left(\varphi_{x}^{\prime}(x, y, 0)\right)^{2}+\left(|u(x, y, 0)|_{x}^{\prime}\right)^{2}\right) d x d y \\
& +\frac{\lambda z}{2 \pi} \int_{-T / 2}^{T / 2} \int_{-T / 2}^{T / 2} 2 x|u(x, y, 0)|^{2} \varphi_{x}^{\prime}(x, y, 0) d x d y \\
& +\int_{-T / 2}^{T / 2} \int_{-T / 2}^{T / 2} x^{2}|u(x, y, 0)|^{2} d x d y .
\end{aligned}
$$

Нетрудно показать, что с учетом выполнения (12) справедливо равенство

$$
\begin{gathered}
\int_{-T / 2}^{T / 2} \int_{-T / 2}^{T / 2}|u(x, y, 0)| \cdot \mid u(x, y, 0)_{x}^{\prime \prime} d x d y \\
=-\int_{-T / 2}^{T / 2} \int_{-T / 2}^{T / 2}\left(|u(x, y, 0)|_{x}^{\prime}\right)^{2} d x d y .
\end{gathered}
$$

Тогда для $m_{20}(z)$ окончательно получим параболическую зависимость от $z$ в виде

$$
\begin{aligned}
& m_{20}(z)=\frac{\lambda^{2} z^{2}}{4 \pi^{2} J_{0}(0)} \int_{-T / 2}^{T / 2} \int_{-T / 2}^{T / 2}\left(\left(\frac{\partial|u(x, y, 0)|}{\partial x}\right)^{2}\right. \\
& \left.+|u(x, y, 0)|^{2}\left(\frac{\partial \varphi_{x}(x, y, 0)}{\partial x}\right)^{2}\right) d x d y \\
& +\frac{\lambda z}{2 \pi J_{0}(0)} \int_{-T / 2}^{T / 2} \int_{-T / 2}^{T / 2} 2 x|u(x, y, 0)|^{2}\left(\frac{\partial \varphi_{x}(x, y, 0)}{\partial x}\right) d x d y \\
& +\frac{1}{J_{0}(0)} \int_{-T / 2}^{T / 2} \int_{-T / 2}^{T / 2} x^{2} I(x, y, 0) d x d y
\end{aligned}
$$

При $\quad T \rightarrow \infty$ и $\lim _{x \rightarrow \infty} I(x, y, 0)=0$ выражение (17) полностью совпадает с [4].

Принципиальным отличием от [4] является необходимость условия (12) при невыполнении которого $m_{20}(z)$ не существует. 
Рассмотрим начальный момент $m_{10}(z)$ :

$$
\begin{aligned}
m_{10}(z) & =\frac{J_{1}(z)}{J_{0}(z)}=\frac{\int_{-\infty}^{\infty} x d x \int_{-\infty}^{\infty} I(x, y, z) d y}{\int_{-\infty}^{\infty} \int_{-\infty}^{\infty} I(x, y, z) d x d y} \\
= & \frac{\lim _{L \rightarrow \infty} \int_{-L}^{L} x d x \int_{-L}^{L} I(x, y, z) d y}{\lim _{L \rightarrow \infty} \int_{-L}^{L} d x \int_{-L}^{L} I(x, y, z) d x} .
\end{aligned}
$$

Выражение для $J_{1}(z)$ аналогично (8) запишем в виде

$$
J_{1}(z)=\frac{1}{\lambda z} \int_{-T / 2}^{T / 2}\left(\lim _{L \rightarrow \infty} \int_{-L}^{L} x \bar{I}\left(x, 0, R_{\bar{y}}, z\right) d x\right) d R_{\bar{y}}
$$

Интегрируя (18) по переменной $x$ и учитывая, что

$$
\begin{gathered}
\int_{-L}^{L} x \exp \left(-i \frac{2 \pi}{\lambda z} x \bar{x}\right) d x=\frac{i \lambda^{2} z^{2}}{2 \pi} \psi^{\prime}(\bar{x}, L) ; \\
\psi(\bar{x}, L)=\frac{1}{\pi \bar{x}} \sin \left(\frac{2 \pi \bar{x} L}{\lambda z}\right),
\end{gathered}
$$

для $J_{1}(z)$ запишем

$$
\begin{aligned}
& J_{1}(z)=\frac{i \lambda z}{2 \pi} \int_{-T / 2}^{T / 2} d R_{\bar{y}} \\
& \times\left(\operatorname { l i m } _ { L \rightarrow \infty } \int _ { 0 } ^ { T / 2 } d R _ { \overline { x } } \int _ { - ( T - 2 R _ { \overline { x } } ) } ^ { T - 2 R _ { \overline { x } } } \left(\bar{\mu}\left(-R_{\bar{x}}, R_{\bar{y}}, \bar{x}, z\right)\right.\right. \\
& \left.\left.+\bar{\mu}\left(R_{\bar{x}}, R_{\bar{y}}, \bar{x}, z\right)\right) \psi_{\bar{x}}^{\prime}(\bar{x}, L) d \bar{x}\right) .
\end{aligned}
$$

Последовательно интегрируя (19) по частям и учитывая, что $\psi(-\bar{x}, L)=\psi(\bar{x}, L), \lim _{L \rightarrow \infty} \psi(\bar{x}, L)=\delta(\bar{x})$, после преобразований получим

$$
J_{1}(z)=-\frac{i \lambda z}{2 \pi} \int_{-T / 2}^{T / 2} \int_{-T / 2}^{T / 2} \bar{\mu}^{\prime}\left(R_{\bar{x}}, R_{\bar{y}}, z\right) d R_{\bar{x}} d R_{\bar{y}}
$$

где

$$
\begin{aligned}
& \bar{\mu}^{\prime}\left(R_{\bar{x}}, R_{\bar{y}}, z\right)=\left[u\left(R_{\bar{x}}+\frac{\bar{x}}{2}, R_{\bar{y}}, 0\right)\right. \\
& \left.\quad \times u^{*}\left(R_{\bar{x}}-\frac{\bar{x}}{2}, R_{\bar{y}}, 0\right) \exp \left(i \frac{2 \pi}{\lambda z} \bar{x} R_{\bar{x}}\right)\right]\left._{\bar{x}}^{\prime}\right|_{\bar{x}=0} .
\end{aligned}
$$

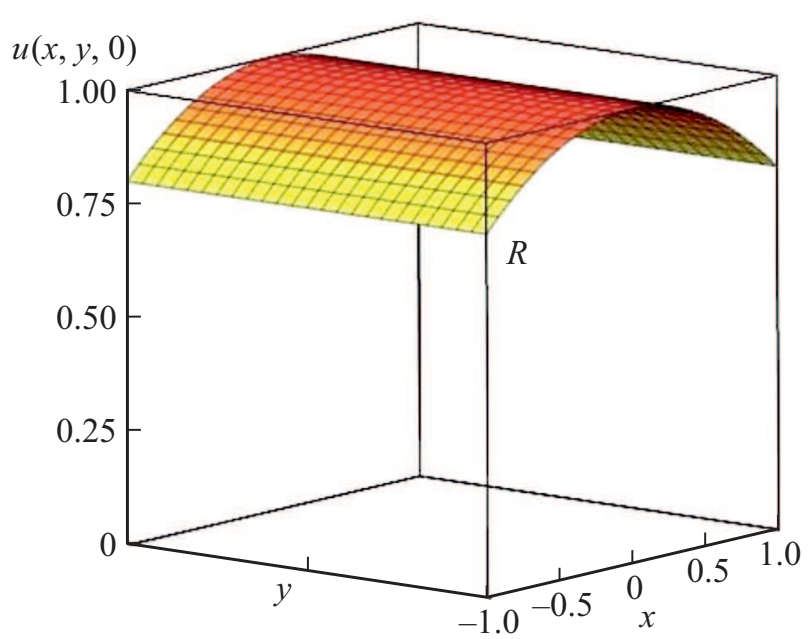

Рис. 2. Квазиравномерное распределение амплитуды поля в плоскости излучателя при $R=0.8 ; T=2$.

Подставляя $(21)$ в $(20)$ и заменяя $R_{\bar{x}}=x ; R_{\bar{y}}=y$, запишем

$$
\begin{aligned}
& J_{1}(z)=\frac{i \lambda z}{4 \pi} \int_{-T / 2}^{T / 2} \int_{-T / 2}^{T / 2}\left(u(x, y, 0) \frac{\partial u^{*}(x, y, 0)}{\partial x}\right. \\
& \left.-\frac{\partial u(x, y, 0)}{\partial x} u^{*}(x, y, 0)\right) d x d y \\
& +\int_{-T / 2}^{T / 2} \int_{-T / 2}^{T / 2} x I(x, y, 0) d x d y .
\end{aligned}
$$

При $T \rightarrow \infty$ последняя формула совпадает с [4]. Таким образом, для момента $m_{10}(z)$ условие $(12)$ не является обязательным.

Применяя (16) для $m_{10}$, окончательно получим

$$
\begin{aligned}
m_{10}(z)= & \frac{1}{J_{0}(0)} \int_{-T / 2}^{T / 2} \int_{-T / 2}^{T / 2} I(x, y, 0) \\
& \times\left(\frac{\lambda z}{2 \pi} \frac{\partial \varphi(x, y)}{\partial x}+x\right) d x d y .
\end{aligned}
$$

Моменты $m_{02}(z)$ и $m_{01}(z)$ вычисляются аналогично.

В заключение рассмотрим пример, иллюстрирующий полученные выводы относительно сходимости вторых моментов.

Рассмотрим квазиравномерное распределение амплитуды, принимающее значение $R$ на границах апертуры $(T / 2, y)$ и $(-T / 2, y),-T / 2 \leq y \leq T / 2$ (рис. 2)

$$
u(x, y, 0)=1-\frac{4(1-R) x^{2}}{T^{2}}
$$

Рассмотрим $m_{20}(z)$. 
Для простоты преобразований, не уменьшающих общность выводов, ограничимся случаем, когда $\lambda z \gg \pi\left(x_{1}^{2}+y_{1}^{2}\right)$ (приближение Фраунгофера), тогда для (3) можно записать

$$
\begin{aligned}
& I(x, y, z)=\frac{1}{\lambda^{2} z^{2}} \int_{-T / 2}^{T / 2} \int_{-T / 2}^{T / 2} u\left(x_{1}, y_{1}, 0\right) \\
& \times \exp \left(-i \frac{2 \pi}{\lambda z}\left(x x_{1}+y y_{1}\right)\right) d x_{1} d y_{1} \int_{-T / 2}^{T / 2} \int_{-T / 2}^{T / 2} u\left(x_{1}, y_{1}, 0\right) \\
& \times \exp \left(i \frac{2 \pi}{\lambda z}\left(x x_{1}+y y_{1}\right)\right) d x_{1} d y_{1} .
\end{aligned}
$$

С учетом четности функции (22) после интегрирования (23) получим

$$
\begin{aligned}
& I(x, y, z)=\frac{\lambda^{2} z^{2} \sin ^{2}\left(\frac{\pi T y}{\lambda z}\right)}{x^{6} y^{2} \pi^{8} T^{4}}\left(\operatorname { s i n } ( \frac { \pi T x } { \lambda z } ) \left(\pi^{2} R T^{2} x^{2}\right.\right. \\
& \left.\left.+2 \lambda^{2} z^{2}(1-R)\right)-\lambda z \cos \left(\frac{\pi T x}{\lambda z}\right)(2 \pi T(1-R) x)\right)^{2} .
\end{aligned}
$$

Нормированный второй момент имеет вид

$$
m_{20}(z)=\frac{J_{2}(z)}{J_{0}(z)}=\frac{\int_{-\infty}^{\infty} d y \int_{-\infty}^{\infty} x^{2} I(x, y, z) d x}{\int_{-\infty}^{\infty} \int_{-\infty}^{\infty} I(x, y, z) d x d y}
$$

Для сходимости интеграла $J_{0}(z)$ достаточно убывание $I(x, y, z)$ по переменным $x$ и $y$ быстрее, чем $1 / x$ и $1 / y$ соответственно, что всегда выполняется.

Для сходимости интеграла $J_{2}(z)$ достаточно убывание $I(x, y, z)$ по переменным $x$ и $y$ быстрее, чем $1 / x^{3}$ и $1 / y$ соответственно. Для $J_{2}(z)$ это условие не выполняется, если $R \neq 0$, т.е. когда РИ на границе не равно нулю. Действительно, в выражении (24) можно выделить слагаемое $\frac{\lambda^{2} z^{2} R^{2} \sin ^{2}\left(\frac{\pi T y}{\lambda z}\right)}{2 \pi^{4} y^{2}}$, интеграл от которого по переменной $x$ расходится.

Заметим, что частный случай, когда в (24) $R=1$, соответствует известной дифракционной картине РИ Фраунгофера на квадратном отверстии [5]

$$
I(x, y, z)=\left(\frac{\lambda z \sin \left(\frac{\pi T y}{\lambda z}\right) \sin \left(\frac{\pi T x}{\lambda z}\right)}{\pi^{2} x y}\right)^{2},
$$

второй момент РИ $m_{20}(z)$ которого расходится. Амплитудное распределение поля при этом равномерно и на границе апертуры излучателя отлично от нуля. Пожалуй, данный пример является самым простым и наглядным подтверждением обсуждаемых результатов.
Если же $R=0$, то

$$
\begin{aligned}
I(x, y, z)= & \frac{4 \lambda^{4} z^{4} \sin ^{2}\left(\frac{\pi T y}{\lambda z}\right)}{x^{6} y^{2} \pi^{8} T^{4}} \\
& \times\left(\lambda z \sin \left(\frac{\pi T x}{\lambda z}\right)-\pi T x \cos \left(\frac{\pi T x}{\lambda z}\right)\right)^{2} .
\end{aligned}
$$

Интеграл $J_{0}(z)$ на основании (15) равен

$$
J_{0}(0)=\int_{-T / 2}^{T / 2} \int_{-T / 2}^{T / 2}\left(1-\frac{4 x^{2}}{T^{2}}\right)^{2} d x d y=\frac{8 T^{2}}{15} .
$$

Тогда, вычисляя $m_{20}(z)$, получим конечное значение второго момента

$$
\begin{aligned}
& m_{20}(z)=\frac{15 \lambda^{4} z^{4}}{2 \pi^{8} T^{6}} \int_{-\infty}^{\infty} \frac{1}{x^{4}}\left(\lambda z \sin \left(\frac{\pi T x}{\lambda z}\right)\right. \\
& \left.-\pi T x \cos \left(\frac{\pi T x}{\lambda z}\right)\right)^{2} d x \int_{-\infty}^{\infty} \sin ^{2}\left(\frac{\pi T y}{\lambda z}\right) / y^{2} d y=\frac{5 \lambda^{2} z^{2}}{2 \pi^{2} T^{2}} .
\end{aligned}
$$

Формула (17), справедливая при $R=0$, приводит к тому же результату. В нашем случае $\lambda z \gg \pi\left(x_{1}^{2}+y_{1}^{2}\right)$ рассматриваем только первое слагаемое

$$
\begin{aligned}
m_{20}(z) & =\frac{\lambda^{2} z^{2}}{4 \pi^{2} J_{0}(0)} \int_{-T / 2}^{T / 2} \int_{-T / 2}^{T / 2}\left(\left(\frac{\partial|u(x, y, 0)|}{\partial x}\right)^{2}\right) d x d y \\
& =\frac{15 \lambda^{2} z^{2}}{16 \pi^{2} T} \int_{0}^{T / 2}\left(\left(1-\frac{4 x^{2}}{T^{2}}\right)^{\prime}\right)^{2} d x=\frac{5 \lambda^{2} z^{2}}{2 \pi^{2} T^{2}} .
\end{aligned}
$$

Заметим, что при доказательстве сходимости моментов также можно использовать асимптотические разложения интегралов [7].

\section{Обсуждение основных результатов и выводы}

Как следует из выражения (12), сходимость вторых моментов обеспечивается при условии равенства нулю пространственного РИ на границах апертуры излучателя, что не гарантируется. Для первых моментов такого ограничения не требуется.

Остановимся на этом подробнее.

Если РИ быстро убывает по мере приближения к краям апертуры излучателя, то можно заменить конечный промежуток $(-T / 2 ; T / 2)$ бесконечным $(-\infty, \infty)$, считая значения РИ за пределами конечного промежутка равными нулю.

Так, в [6] на примере пучка с осесимметричным гауссовым распределением амплитуды поля 
$u(r)=\exp \left(-r^{2} / w^{2}\right)$ и радиусом ограничивающей диафрагмы $T / 2$ показано, что при $T / w \geq 4.3$ результаты расчета практически полностью соответствуют случаю распространения неограниченного гауссова пучка.

Это значит, что при замене конечного промежутка интегрирования бесконечным быстро убывающее РИ с высокой степенью точности может быть заменено РИ, рассчитанным на бесконечном промежутке. Однако данное заключение, справедливое для РИ, не применимо к его вторым моментам, они расходятся, так как нет оснований считать интенсивность на границах апертуры равной нулю, и даже близость к нулю не обеспечивает сходимость.

В таком случае существует путь вычисления вторых моментов с учетом ограниченной апертуры или чувствительности приемного измерительного устройства, обеспечивающий всегда их сходимость [8], но при этом результат определения характеристик начинает зависеть от параметров этого устройства. Указанный фактор непосредственно сказывается на результатах сравнения различных излучателей, о чем также упоминается в [2].

Таким образом, остаются вопросы к корректному применению упомянутых характеристик, ответы на которые и подробное обсуждение не является предметом настоящей работы и в большей степени касаются метрологов.

Здесь же ставилась задача обратить внимание на существующую в оптике проблему результата замены бесконечной апертуры излучателя реальной апертурой с конечными размерами при определении пространственно-энергетических характеристик лазерного пучка, основанных на вторых моментах РИ.

\section{Конфликт интересов}

Авторы заявляют, что у них нет конфликта интересов.

\section{Список литературы}

[1] ISO 11146:2005 Lasers and laser-related equipment - Test methods for laser beam widths, divergence angles and beam propagation ratios - Part 1-3.

[2] Ананьев Ю.А. // Опт. и спектр. 1999. Т. 86. № 3. C. $499-502$.

[3] ГОСТ 26086-84 Лазеры. Методы измерения диаметра пучка и энергетической расходимости излучения. М.: Издательство стандартов, 1985.

[4] Власов С.Н., Петрищев В.А., Таланов В.И. // Радиофизика. 1971. T. XIV. № 9. C. 1353-1363.

[5] Гудмен Дж. Введение в фурье-оптику. М.: Мир, 1970. $364 \mathrm{c}$.

[6] Климков Ю.М. Прикладная лазерная оптика. М.: Машиностроение, 1985. $124 \mathrm{c}$.

[7] Федорюк М.В. Асимптотика: Интегралы и ряды. М.: Наука, 1987. 544 c.

[8] Райщин А.М. // Измерительная техника. 2013. № 8. C. 23-27; Raitsin A.M. // Measurement Techniques. 2013. V. 56. N 8. P. $868-875$. 